\title{
EVALUATING TWO WAYS TO TRAIN SENSITIVITY TO ECHOES TO IMPROVE ECHOLOCATION
}

\author{
Laurie M. Heller, Arley Schenker, Pulkit Grover, Madeline Gardner, Felix Liu \\ Carnegie Mellon University \\ Pittsburgh, Pennsylvania
}

\begin{abstract}
We investigated whether training sighted individuals to attend to information in echoes could improve their active echolocation ability. We evaluated two training techniques that involved artificially generated sounds. Both artificial techniques were evaluated by their effect on natural echolocation of real objects with self-generated clicks. One group trained by discriminating sounds presented over headphones in the lab. The lateral displacement or distance of the echo was varied in a staircase procedure. The second training group used an echolocation app on a smartphone. They navigated a maze by using echo cues presented over earbuds. The echo cues had 3D audio virtual reality cues. Participants in the control condition did not improve but the majority of participants who trained did improve. The lab training is labor intensive whereas the app training was self-guided and convenient. This has implications for training methods aimed at echolocation that might ultimately be useful for navigation by visually impaired individuals.
\end{abstract}

\section{INTRODUCTION}

Visually impaired people use sound and other sensory cues to navigate, compensating for their loss of vision. In rare cases, they develop the skill of echolocation, in which users self-generate tongue clicks in order to gain information about the surrounding environment. These source, or referent, clicks reflect off of surrounding objects and travel back to the ears, allowing echolocators to perceive information about the location and characteristics of the reflecting object. Early research on echolocation most often concerned bats and dolphins; however, there is a recent increase in effort to study human echolocation and other navigational techniques to inform navigation devices for the visually impaired population [1].
Echolocation using tongue clicks does not occupy a hand (as does a cane, guide dog, or most other navigational devices). The only devices necessary are the head, mouth, and ears, so echolocation is neither expensive nor unwieldy. Echolocators have substantial control over their own tongue clicks. They can also move their head in order to emit clicks in varying directions, which allows them to more precisely detect objects on their left or right. For these reasons, training to use echolocation has the potential to help visually impaired people with normal hearing to navigate more independently. Current systems, some using auditory virtual reality, exist in laboratories for echolocation study purposes [2], [3]. However, it is desirable to make echolocation training easier and more accessible. For this reason, we assessed two methods for training people to discriminate artificial echo information and we measured its success with natural echolocation. We used sighted participants for this preliminary test in order to refine the methods.

\section{ECHO ACOUSTICS AND PSYCHOACOUSTICS}

Echolocation, while a potentially useful skill, is not in common use as a navigational tool. First of all, echoes are subject to masking in loud environments as echoes are very low amplitude sounds. They are acoustically complex and depend on many factors in the physical environment such as how reflective the surrounding material is. Second, echoes are difficult to use because humans normally suppress the locations of environmental echoes.

\subsection{The precedence effect}

First described by Wallach and colleagues, the precedence effect describes the localization of two sounds occurring in short succession. When two similar sounds with a small delay between them are played from different locations, both are heard to have come from the location of the first sound [4]. This gives precedence to 
the location of the first sound. As an echo is simply a quieter version of a referent click, the precedence effect is detrimental to echolocation. People can be trained to be more sensitive to echo information after many hours of training in a laboratory task [5]. The precedence effect has been quantified in an echolocation context by Wallmeier and colleagues, where participants localized echoes reflected by a single sound reflector and the leading and lagging of two reflectors [6].

\subsection{Acoustic cues to location}

When a click is emitted, it bounces off the objects and surfaces in the environment that do not absorb it. Many echoes return to the ears, and several computations can occur to determine information about the location of the sound. For instance, the closest object to the observer will return the first echo. The time between the outgoing (referent) click and the echo will be directly related to the distance of the reflecting object and the speed of sound. Although echo delay is a reliable cue to distance, there are other less reliable cues to distance such as frequency content or echo level relative to the source level. Sound loses intensity the farther it travels [7]. These cues can be simulated by modeling the physical environment, including the absorption characteristics of all surfaces. For the lateral and vertical localization of echoes, there are even more cues. A sound coming from the left side of the listener, for example, arrives at the left ear before arriving at the right ear [7]. These interaural time differences (ITDs) are on the order of microseconds. As sound travels it can also lose intensity at one ear relative to the other, depending on the frequency and direction of the sound. These interaural level differences (ILDs) are another source of information used to localize sound information. Furthermore, individuals' outer ears, or pinnae, are uniquely shaped to amplify certain frequencies and attenuate others, depending on the direction of the sound source. Both the head and pinna come together to form a complex direction-dependent filter [7]. This filtering of incoming sound is known as a head-related transfer function (HRTF). These computations must occur simultaneously for all incoming sounds, and in a reverberant environment, it can be difficult to localize the various overlapping sounds.

\subsection{Training to use echo information}

Surprisingly, given all these acoustic challenges, both blind and sighted individuals can be trained to use echoes to glean information about objects around them. Studies comparing the two populations often compare blind expert echolocators, blind non-echolocators, and sighted individuals. Teng and Whitney showed that after four hours of training, sighted participants could use self-generated clicks to discriminate the size of an object as well as a blind expert echolocator could [8]. Additionally, Schenkman and Nilsson showed that blind participants were better able to report a sound that had been recorded in the presence of a reflecting object, compared to sighted participants; however, both blind and sighted participants could perform the task after training [9]. Finally, sighted participants have been shown to use echoes to discriminate the distance of objects after only one hour of training [10].

Although it has been shown that both blind and sighted individuals can perform simple echolocation tasks, the majority of them have been trained under the same conditions as the test. Even though lab training for specific focused tasks can succeed in a few hours, the training process for individuals who use echolocation on a daily basis for navigation can take years. We therefore searched for a way to make the initial learning process easier and less task-specific. We hypothesized that individuals would be able to learn to discriminate echoes if they were enhanced. As echolocation has been shown to have functional benefits for its users, the process by which individuals become proficient echolocators would ideally be as easy as possible [11]. We amplified the echoes above realistic levels and we decluttered the sound environment. In this study, we investigated whether asking sighted individuals to discriminate these artificially enhanced echoes in a variety of discrimination tasks could help them to actively echolocate large objects using natural mouth clicks. We used two methods for training, one involving a smartphone application that was a game called EchoExplorer $^{\mathrm{TM}}$ (described in more detail in [12]). The other training method was the traditional psychophysical technique of training using a staircase adjustment method while doing two-interval forced-choice discrimination of both distance and lateral position.

\section{METHODS}

\subsection{Design}

Thirteen naive sighted participants were tested, six in the App condition (average age 21.8), five in the Lab condition (average age 19.1), and two in the Control condition (average age 20.5). All participants were first 
pre-tested on real-world discrimination of board positions using their own clicks for echolocation. Next, the Lab and App participants completed 15 hours of echo discrimination training prior to being post-tested in the same task. The Control group did no training but waited a similar amount of time between pre and post tests. The methods of training for the App and Lab groups were different and are described in separate sections below. Written consent was obtained from all participants, participants were paid, and the study protocol was approved by the Carnegie Mellon University Institutional Review Board (IRB).

\subsection{Echolocation pre and post-tests}

In the pretests and posttests we asked participants to indicate the locations of objects but did not give them any feedback; additionally, we discouraged outside research on human echolocation between pre and post tests. During pre and post-tests, blindfolded participants made clicks to locate a board while seated in the center of a large, otherwise empty room. We told them how to make palatal clicks but gave no training or feedback. Across trials, a large board was located in one of 4 lateral angles and one of 3 distances. Participants localized boards with either a fixed head position or with free head movement.

The $1 / 2$ " thick melamine board measured 24 " by 48 " and was reinforced throughout its length by a $2 \times 4$ " wood beam to enhance its rigidity. The board was held vertically and a 1" foam pad was added to its bottom edge to help reduce the sound produced by setting the board down. The board was placed in one of 12 locations relative to the front of the participant's head: one of three distances $(0.9,1.8$ or $2.7 \mathrm{~m})$ and one of four lateral angles (45 or 90 degrees to the right, 45 or 90 degrees to the left). Two trials at each possible location yielded 24 test trials that were presented in one of four counterbalanced random orders. In order to mask any subtle sounds made by experimenters placing the board, participants listened to white noise through earbuds during trial set-up and take-down. At the start of each session, the white noise level was set empirically to be at a comfortable level that successfully masked the sound of experimenter asking the participant a question at a normal conversational level.

The participant was blindfolded while seated in the center of an unfurnished 28' x 56' carpeted room with fabric-covered walls. The experimental trials were preceded by one practice trial. At the start of a trial, the participant placed earbuds in their ears and listened to white noise. The experimenter stood at a central starting location, 2.7 meters directly in front of the participant (holding the board) and announced loudly that the next trial was starting. At that point the listener began counting silently up to 10 . During that time, the experimenter with the board moved the board into position and stood behind the board. The participant stated "ready" before taking their earbuds out. Holding their head level and facing directly ahead, they made 10 clicks with their mouth. They then guessed the direction and distance of the board (e.g. 45 right, 2 for medium distance). Immediately after this, they freely moved their head to the left and right while clicking 10 more times. After this, they made another estimate as to the board's direction and distance. Participants put the earbuds back in, listening to noise while the board was moved back to the center starting location before the next trial began.

\subsection{Game training}

\subsubsection{Participants}

After pretesting on echolocation, six naive users were trained using a beta version of the EchoExplorer ${ }^{\mathrm{TM}}$ app [12] for 15 hours. This game was an app on a smartphone that was designed for this purpose and could be played at the participant's convenience. This training duration was chosen because video game experiments suggest that 15 hours is sufficient to induce some measurable changes in transferred skills [13]. All of these participants had normal hearing according to in-lab audiograms performed with a MAICO MA41 audiometer.

\subsubsection{Procedure}

The goal of the game was to provide a training platform for learning echolocation. We designed a game that requires the user to navigate through various mazes using simulated echoes. An avatar is used to represent the current location of the user in the maze. Although it is possible to see a depiction of the maze and the avatar in a debugging mode, all game play was done with a blank screen (no visual information). At any point in time the user can instruct the application (e.g. by tapping on the touchscreen) to generate echoes based on the current location of the avatar within the maze. The application generates a click followed by the echoes that convey spatial information through echo delays, ITD, and/or ILDs (see Section 2). For instance, if the user is facing a close wall straight ahead, the referent click and the resulting echo will be heard in quick succession. If on the other hand, the wall is much further from the 
user's current location, the difference between the time when the reference click is heard and when the corresponding echo is heard will be much larger.

We used the high-resolution acoustics modeling program Odeon, a room acoustic simulation and measurement software, in order to artificially create echoes of clicks based on the room shape. This program generates acoustic cues based on the shape of the maze: for example, the time delay between the emitted sound and the echo provides a reliable cue as to the distance of nearby obstacles. The spatial location of the nearby obstacles is further indicated by the acoustic effects of the head in the path of the echoes. (HRTF 58 was used from the CIPIC database [14] because it was measured from a head with anthropometric feature closest to the average of the CIPIC database and has been validated with naive users in favorable comparison to KEMAR [15]. However, combinations of HRTFs may have different acoustic properties than individual HRTFs and may be worth using in the future [16]).

After creating a set of echoes in Odeon to simulate hallways ending in all the possible junctions (deadend, elbow left, elbow right, t-junction, and stairwells) we boosted the level of all the echoes by the same amount to increase their audibility while keeping the outgoing reference click at its original level. This helped users distinguish between the subtle changes in echoes as they moved through a hallway. After pilot testing, the echoes were boosted $21 \mathrm{~dB}$ at the start of the game. Every 15 levels in the game, the echo boost was decreased by $2 \mathrm{~dB}$ until users indicated that the game was too difficult to play. This lower limit occurred at or above an echo boost of $7 \mathrm{~dB}$ or all users.

Auditory cues were given after each move gesture just to indicate that the motions had been accomplished, but no correct/incorrect feedback was given for individual motions unless that motion caused the avatar to crash into a virtual wall of the maze, in which case, a "crash" sound was played. Implicitly, the absence of a crash sound after a motion indicated that it was a safe move, but nothing indicated whether or not the avatar was heading in the correct direction. However, if the avatar returned all the way to the start of the maze, an auditory warning was played.

\subsection{Traditional Psychoacoustic Training}

\subsubsection{Participants}

After pretesting with echolocation, we trained 5 sighted college students in the laboratory for 15 hours with artificial sounds. All participants were verified to have normal hearing except for one participant who had a mild low-frequency hearing loss in one ear ( $25 \mathrm{~dB} \mathrm{HL})$ from 250 to $1000 \mathrm{~Hz}$; who exhibited normal sensation levels and normal interaural discrimination thresholds using our broadband click stimuli.

\subsubsection{Stimuli}

Palatal mouth referent clicks, recorded by an undergraduate research assistant, were used as a foundation for the stimuli. These clicks were recorded using Roland CS-10EM binaural microphones in an empty IAC sound-attenuating chamber in which the walls and ceiling were covered with 4-in. Auralex foam wedges to reduce echoes. Over 50 clicks were recorded, but only the 17 clicks with waveforms similar to the ideal palatal clicks described by Rojas were used [17]. A custom echo generation program, written in Matlab, was applied to each of these clicks to generate realistic echoes. The echo was generated by adding a copy of the referent click at the appropriate delay corresponding to the distance of the reflecting object. The referent sound travels to the reflecting object and back to the listener as an echo, so it travels twice the distance between the listener and the object. Then, using a speed of sound of $343 \mathrm{~m} / \mathrm{s}$, an echo from an object $5 \mathrm{~m}$ away, for example, would occur 29 milliseconds $((2 * 5) \mathrm{m} / 343 \mathrm{~m} / \mathrm{s})$ after the onset of the referent.

When tracking on lateral position, ILDs were implemented in the left and right channels by attenuating one channel according to the angle and distance of the reflecting object. The maximum ILD, used when an object was $90^{\circ}$ to the left or right, was $10 \mathrm{~dB}$. ILDs for angles between $0^{\circ}$ and $90^{\circ}$ were $((1 / 9) *$ angle $) \mathrm{dB}$. The overall level of the echo decreased in both channels by an additional $6 \mathrm{~dB}$ each time distance was doubled relative to $1 \mathrm{~m}$. During some tracks interaural time delay (ITD) was manipulated instead of ILD. (Those data obtained with ITDs are not presented here due to incomplete data sets.) Determination of the echo levels at which the tasks could be performed at a medium difficulty level for the average person occurred during pretesting. In subsequent staircases, echo levels were reduced as needed to keep thresholds similar over time. Echoes were generated at 10-degree intervals between $-90^{\circ}\left(90^{\circ}\right.$ to the left) and $+90\left(90^{\circ}\right.$ to the right $)$. In addition, echoes were generated at distances between 1 and 5 meters at 0.5 -meter increments. The referent click was always presented at the same level, $30 \mathrm{~dB}$ SL (sensation level). Within one trial, the reference click was the same for both intervals; the only difference 
between the intervals was the timing and level of the echo, calculated relative to the referent click, that accompanied each click. Between trials, clicks were chosen randomly from the 17 clicks described previously.

\subsubsection{Procedure}

After providing written consent, participants were informed about the structure of the tasks and were given the opportunity to ask questions. They were also instructed not to focus on any one cue in the stimuli and to close their eyes during the experiment. They performed the experiment while seated at a computer in the aforementioned listening chamber. Participants listened to the stimuli through Sennheiser HD 600 headphones.

There were two main tasks: distance and left/right discrimination. All participants performed the distance task first within each of the 15 hour-long test sessions. Each of these tasks was a 2-interval forced choice (2IFC) task in which the order of the intervals was randomized. During the distance task, each interval of a trial contained a referent click, which was centered (no ILDs were applied) and whose channels were normalized relative to each other, followed by an echo generated according to the aforementioned parameters. The angle of echoes in the distance task was kept constant at $0^{\circ}$. Participants discriminated a click with a close echo from a click with a far echo and reported which interval, 1 or 2 , contained the closer echo by pressing the corresponding key. During the left/right task, the echo always had a simulated distance of $1 \mathrm{~m}$ whereas the angle varied between $-90^{\circ}$ and $+90^{\circ}$ at 10-degree increments. In the left/right condition, participants reported whether the echoes in the two intervals moved from right to left or from left to right by pressing the 1 or $2 \mathrm{key}$, respectively.

During each trial of the 2IFC tasks, participants were shown a sentence reminding them of the correct key presses. (For example, in the distance condition: "Which click contained the closer echo? (1 2)".) A trial consisted of two clicks separated by $500 \mathrm{~ms}$ of silence. For example, a trial in the distance condition could contain a click with an echo from $1 \mathrm{~m}$ away, followed by $500 \mathrm{~ms}$ of silence, followed by a click with an echo from $4.5 \mathrm{~m}$ away. Prerecorded verbal feedback was given (e.g. "correct!"). The next trial was determined using a three-down-one-up staircase paradigm [18]. The staircase paradigm allowed for the determination of a threshold at which participants responded accurately to about $78 \%$ of the trials. At the beginning of all three conditions, the staircases started at the easiest level (distance: 5m, lateralization and left/right: 90 degrees). If participants correctly answered three trials in a row, the subsequent trial increased in difficulty by one level. If participants incorrectly answered a single trial, the subsequent trial decreased in difficulty by one level. The track ended after 11 reversals were observed or if the track lasted for over 70 trials without 11 reversals. Here, reversals are defined to be points during the track where participants answered correctly three times after answering incorrectly on the previous trial, or points where participants answered incorrectly once after answering correctly on the previous trial. Participants performed 2-5 tracks per condition per test session. A condition average for each participant was calculated by computing the average of each of that participant's tracks. If the track did not contain 6 reversals or if the participant performed more than 3 trials at the easiest level during any given reversal in the last 6 reversals, that track was not included in the participant's threshold calculation. The staircase adjusted the distance (in depth, or angular distance) between the two intervals of a trial and determined a threshold. After each track, performance was reviewed by an experimenter. If the participant's performance was good, the level of the echo was decreased in the next track. In this way, the experimenter aimed to keep the threshold relatively constant while gradually decreasing the echo level over time.

\section{RESULTS OF GAME PLAY VS. PSYCHOACOUSTIC TRAINING}

\subsection{Learning during training}

Using the EchoExplorer ${ }^{\mathrm{TM}}$ game, we measured number of crashes into walls per level, number of echoes requested per level, number of steps taken per level, and active time per level. Because echo level did decrease by $2 \mathrm{~dB}$ every 15 levels after the tutorial, in order to look for learning effects, we pulled out performance at a few echo levels to compare the number of crashes per level as training went on. The maze level was cycled through a few times so that we were able to compare performance at similar echo/maze levels over time. Figure 1 shows log fits to the average number of crashes per level as a function of the sequential time of play of each level. Crashes were higher initially for the earliest level with the $21 \mathrm{~dB}$ boost because it was the first level encountered but, as expected, the asymptotic 


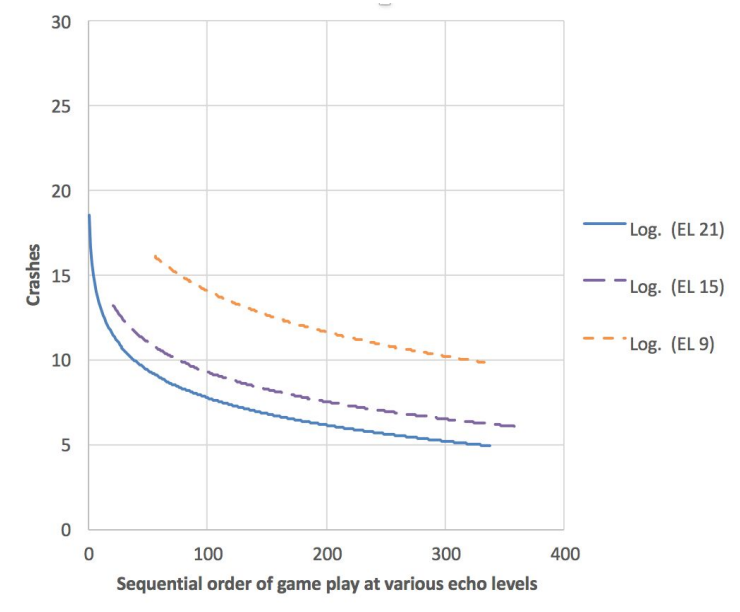

Figure 1. Number of times crashing into maze walls as a function of the sequential order in which the level was completed. The parameter is the level boost of the echo in $\mathrm{dB}$, either 9,15 or $21 \mathrm{~dB}$. The average data across all App participants are fitted with Log functions.

performance level was best for this condition. Even at the most difficult levels, the echo was still boosted beyond what would typically occur in a real hallway.

There was evidence of learning during the lab training. Across participants in the Lab group, the average simulated distance that supported $78 \%$ discrimination from a $1 \mathrm{~m}$ distance was $4.45 \mathrm{~m}$ (SD 0.9). Figure 2 shows the echo level required for discrimination of distance as a function of training hour for four participants. (Varying echo level data were not available for the fifth participant due to a procedural error). Improvement (measured as a decrease in echo level) ranged from 4 to $12 \mathrm{~dB}$ over time. Across participants, the average simulated angle difference that supported $78 \%$ correct discrimination between right and left lateral positions was 38.4 degrees (SD 9.9). Figure 3 shows the echo level required for discrimination of lateral position (in the left/right task) for all 5 participants in the Lab group. In both graphs, the echo level required decreased over time. The echo was still boosted beyond what would typically occur in natural conditions, but by the end, all participants could reliably discriminate the echo when its level was lower than the source level.

\subsection{Improvement in echolocation}

Discrimination of lateral position and distance was evaluated in both a pretest and posttest. Dprime was the measure of sensitivity to the different possible locations of the board [19]. The four lateral positions $(-90,-45,45$,

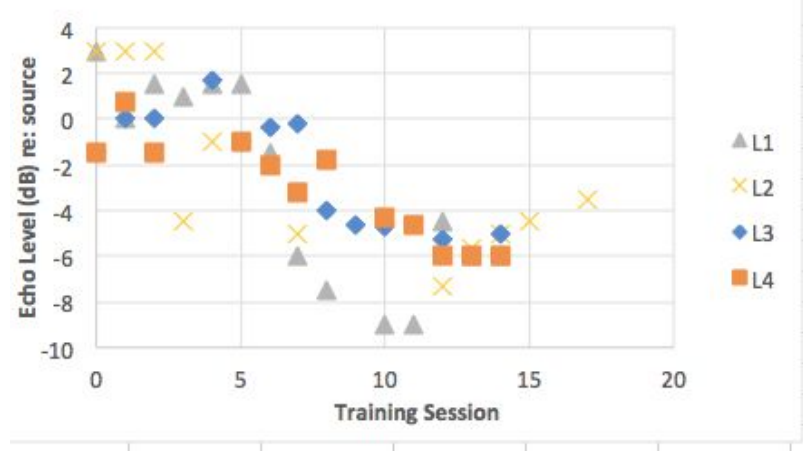

Figure 2. Echo level used relative to the initial outgoing click in order to support average threshold performance on distance discrimination as a function of hours of training in the lab (for individuals in the Lab group).

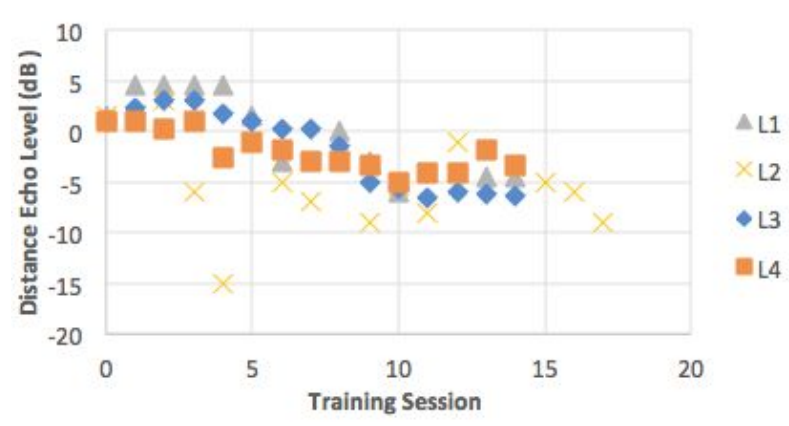

Figure 3. Echo level used relative to the initial outgoing click in order to support average threshold discrimination of left/right lateral position as a function of hours of training in the lab (for the Lab group).

and 90) yielded a chance level of $25 \%$ which would be equivalent to a d' of 0 for a four-alternative forced-choice task. The three possible distances $(0.9,1.8$ and 2.7 meters) yielded a chance level of $33 \%$ with a d' of 0 for a three-alternative forced-choice task.

When head position was fixed, there was modest sensitivity to lateral position with an average d' of 0.10 at pretest and 0.43 at posttest, with 9 of 11 trained participants showing improvement. When the head was moved freely, the average lateral position discrimination was 0.15 at pretest and 0.64 at posttest, with 10 of 11 participants showing improvement. For the head moving condition, post test d' versus pretest d' is shown for all observers in Figure 4. No change in performance would be implied by the dashed line, whereas improvement is indicated by all data points above that line. The average sensitivity was relatively low given that a d' value of 1 is typically considered threshold discrimination, similar to the $78 \%$ correct 


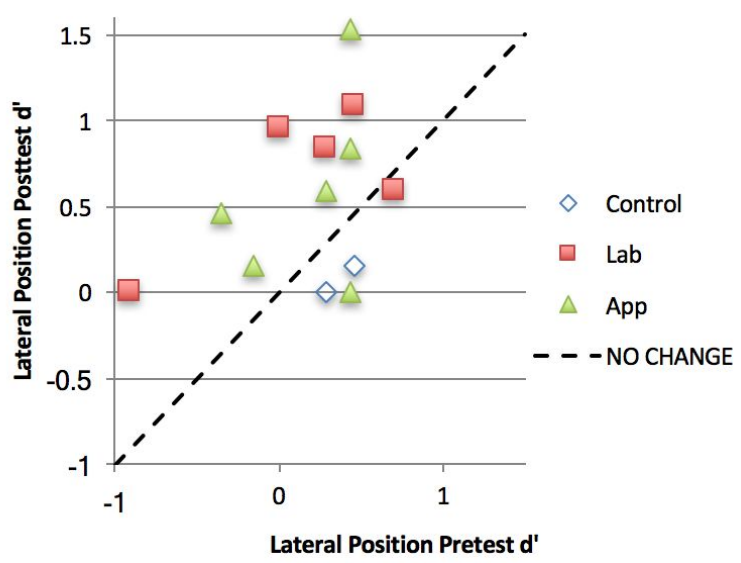

Figure 4. Post test d' (sensitivity) to lateral position as a function of Pre test d' in a real-world discrimination task when the head was moved freely. Control participants (open diamonds) did not have any training between tests. Lab participants (red squares) and App participants (green triangles) were trained in the lab, or used the app, respectively, for 15 hours between tests. Data points above the dashed line indicate improvement.

value that we targeted in the lab training. Nonetheless, the average improvement in d' $(0.30$ across conditions, 0.11 for fixed, 0.49 for moving) was reliably greater than zero (tested by a mixed ANOVA using a between subject factor of training group and within-subject factors of fixed vs moving head and pre vs post test $(F(1,4)=63, p<.001)$. There no significant main effect of the type of training (Lab and App groups) and no effect of the fixed and moving head conditions, nor were there any interactions. Note that the condition in which the head moved also benefited from more observations because it came after the fixed condition, so we would not draw conclusions about head movement per se from this result. Among the $44 \%$ of post-test lateral position trials in which trained participants changed their answers between the fixed and head moving conditions, they changed from an incorrect to a correct answer $42.6 \%$ of the time (whereas chance would be $33 \%$ ). The two control participants displayed pretest dprimes within the middle of the range of all other participants and did not improve their sensitivity between pre and post tests.

Average discrimination of distance was low in the $3 \mathrm{afc}$ task, but reliably above chance (average of $\mathrm{d}^{\prime}=0.215$, with $95 \%$ confidence intervals for all conditions above zero). There was no learning of distance between pre and post tests as indicated by an ANOVA with factors of training group, pre/post test and fixed/moving head. Average distance d' did not improve between pretest $(0.22)$ and posttest $(.21)$ and this did not interact with training group or head movement. Data were missing from one participant in the lab group in the fixed head distance discrimination; the resulting App group did have a lower average d' $(0.12)$ than the Lab group (0.31) $(\mathrm{F}(1,8)=6.13, \quad \mathrm{p}<.038)$, but because distance discrimination did not significantly change at post-test, this group difference was inconsequential for characterizing training effects. Dprime for distance was not reliably lower when the head was fixed (0.19) compared to moving $(0.24)$ nor did it interact with any other factors.

\section{CONCLUSION AND DISCUSSION}

At the outset, there were many factors working against the possibility that our first attempt to train people with this game would produce measurable echolocation benefits in the real world. Among these factors were the fact that this was a beta test of an app; our participants were sighted; the training conditions did not match the test conditions in terms of the reflector locations; the referent clicks were not the participant's own clicks; the HRTFs were not emitted by the participant; and the training echoes were louder than they are in real life. For this reason, we find the small reliable benefits that we measured to be encouraging.

If sighted users can gain some small benefit from this training, the next step after further refinement would be to test blind users. At this point, we can conclude that training with artificial sounds - sounds that do not require the participant to emit clicks or move - can be effective in improving echolocation with self-generated clicks in the real world. At the start of this study it was not known whether a game such as ours could produce a benefit.. Note that this new finding of transfer is distinct from showing that training on echolocation in the real world improves echolocation in the real world. Even so, locating a board while moving one's head is only one small step towards using echolocation to navigate while moving in a realistic environment. Although it is likely that our artificial training may be less effective than if the same amount of time were spent navigating in the real world while exclusively using mouth clicks, both of our training methods have potential advantages, especially when many hours of solo training are required. Our methods allow the use of enhanced echoes, and our data show that this enhancement was required to achieve reliable discrimination at the start for all 
participants. We saw that learning in the lab using a standard psychoacoustic method was not substantially superior to learning with the app. However, a key advantage to training with the game is that it is under the user's control and can be used at their convenience; it therefore is more accessible and practical than the customized psychoacoustic training method in the lab. It should also be pointed out that the EchoExplorer ${ }^{\mathrm{TM}}$ game was tested as a beta version and is not yet optimized. Therefore, we find these experimental results encouraging for the future of using games to learn new ways to use sound for navigation.

\section{ACKNOWLEDGMENT}

We thank Sarah Kwan, Tejal Kudav, Kiran Matharu, Jacqueline Hon, Jessica Kwon, Aaron Schwartz, Chieko Asakawa, Catherine Getchell, participants at the Blind and Vision Rehabilitation Services of Pittsburgh, and Art Rizzino for helpful discussions, feedback and pointers. We also thank Google Inc., the NSF Center for Science of Information (CSoI), CMU's SURG program, and the NSF REU program for their generous support.

\section{REFERENCES}

[1] A. J. Kolarik, S. Cirstea, S. Pardhan, and B. C. J. Moore, "A summary of research investigating echolocation abilities of blind and sighted humans," Hearing Research, vol. 310, pp. 60-68, 2014.

[2] B. F. G. Katz and L. Picinali, "Spatial audio applied to research with the Blind.," in Advances in Sound Localization, 2011, pp. 225-250.

[3] D. Pelegrin-Garcia, M. Rychtáriková, C. Glorieux, and B. F. G. Katz, "Interactive auralization of self-generated oral sounds in virtual acoustic environments for research in human echolocation," in Proceedings of Forum Acusticum 2014, 2014.

[4] H. Wallach, E.B. Newman, and M. R. Rosenweig, "The precedence effect in sound localization," The American Journal of Psychology, vol. 62(3), 315-336, 1949.

[5] K. Saberi, and J. V. Antonio, "Precedence-effect thresholds for a population of untrained listeners as a function of stimulus intensity and interclick interval," $J$. Acoustical Soc. Am., vol. 114, pp. 420-429, 2003.

[6] L. Wallmeier, N. Geßele, and L. Wiegrebe, "Echolocation versus echo suppression in humans," Proceedings of the Royal Society B., vol. 280,
$20131428,2013$.

[7] B. C. J. Moore, An Introduction to the Psychology of Hearing, Sixth Edition. Bingley, UK: Emerald Group Publishing Limited, 2012.

[8] S. Teng, and D. Whitney, "The acuity of echolocation: Spatial resolution in the sighted compared to expert performance," Journal of Visual Impairment and Blindness, vol. 105(1), pp. 20-32, 2011.

[9] B. N. Schenkman, and M. E. Nilsson, "Human echolocation: Blind and sighted persons' ability to detect sounds recorded in the presence of a reflecting object," Perception, vol. 39, pp. 483-501, 2010.

[10] A. Tonelli, L. Brayda, and M. Gori, "Depth echolocation learnt by novice sighted people," PLoS One, vol. 11, no. 6, pp. 1-14, 2016.

[11] L. Thaler, "Echolocation may have real-life advantages for blind people: an analysis of survey data," Frontiers in Psychology, 2013, vol. 4(98), eCollection.

[12] W. Wu, R. Morina, A. Schenker, A. Gotsis, H. Chivukula, M. Gardner, F. Liu, S. Barton, S. Woyach, B. Sinopoli, P. Grover, and L. M. Heller, "EchoExplorer" : A game app for understanding echolocation and learning to navigate using echo cues," in ICAD Proceedings 2017, 2017.

[13] C. S. Green, D. Bavelier, "Action video game training for cognitive enhancement," Current Opinion in Behavioral Sciences, vol. 4, pp. 103-108, 2015.

[14] R.V. Algazi, R.O. Duda, D.M. Thompson, C. Avendano, The CIPIC HRTF Database, IEEE ASSP Workshop on Applications of Signal Processing to Audio and Acoustics In WASSAP '01 (2001).

[15] E. De Sena, N. Kaplanis, P.A. Naylor, T. van Waterschoot, "Large-scale auralised sound localisation expe riment," AES 60th International Conference, 2016.

[16] L. S. R. Simon, N. Zacharov, and B. F. G. Katz, "Perceptual attributes for the comparison of head-related transfer functions," J. Acoust. Soc. Am., vol. 140, no. 5, pp. 3623-3632, 2016.

[17] J. A. M. Rojas, J. A. Hermosilla, R. S. Montero, and P. L. L. Espi, "Physical Analysis of Several Organic Signals for Human Echolocation: Oral Vacuum Pulses," Acta Acustica United with Acustica, vol. 95, pp. 325-330, 2009.

[18] M. R. Leek, "Adaptive procedures in psychophysical research," Perception \& Psychophysics, vol. 63(8), pp. 1279-1292, 2001.

[19] N.A. Macmillan and C.D. Creelman, Detection Theory: A Users Guide, 2nd Ed. Psychology Press, 2004.

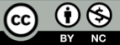

This work is licensed under Creative Commons Attribution - Non Commercial 4.0 International License. The full terms of the License are available at http://creativecommons.org/licenses/by-nc/4.0/ 